\title{
Pd-catalyzed intramolecular aminofluorination of allylic sulfamides
}

\author{
Jiashun Cheng, Pinhong Chen, Guosheng Liu* \\ State Key Laboratory of Organometallic Chemistry, Shanghai Institute of Organic Chemistry, Chinese Academy of Sciences, Shanghai 200032, China
}

\section{A R T I C L E I N F O}

\section{Article history:}

Received 19 May 2014

Accepted 9 June 2014

Published 20 January 2015

Keywords:

Palladium-catalyzed

Aminofluorination

Alkene

Fluorinated 1,3-diamine

6-Endo cyclization

\section{A B S T R A C T}

A facile Pd-catalyzed intramolecular aminofluorination reaction of allylic sulfamides was developed that can be used to prepare fluorinated 1,3-diamine derivatives. Acetic acid was essential for regulation to give the major 6-endo cyclization products.

(C) 2015, Dalian Institute of Chemical Physics, Chinese Academy of Sciences. Published by Elsevier B.V. All rights reserved.

\section{Introduction}

The introduction of fluorine into organic molecules frequently leads to dramatic changes of their properties, such as solubility, metabolic stability, and bioavailability [1,2]. Among these organofluorine molecules, fluorinated heterocycles are extensively used as important building blocks for the synthesis of anticholinergic, antiemetic, and antispastic drugs as well as enzyme inhibitors [3]. Thus, the efficient synthesis of these fluorinated heterocycles has attracted much attention [4-13].

Palladium-catalyzed difunctionalization reactions are a powerful strategy for the direct synthesis of vicinal substituted molecules from simple olefin feedstock. Among these reactions, oxidation of the alkyl-Pd intermediate generated from the initial nucleopalladation of alkenes is essential for this difunctionalization, in which a hypervalent palladium complex has been proposed to respond to the $\mathrm{C}-\mathrm{X}$ bond formation $[14,15]$. In 2009 , our group reported the first palladium-catalyzed intra- molecular aminofluorination of unactivated alkenes, and $\mathrm{C}-\mathrm{F}$ bond formation was obtained through the oxidative cleavage of the alkyl-Pd bond with a AgF/PhI(OPiv) 2 system. The reaction gave a series of fluorinated piperidine products with high regioselectivity [15]. Recently, we found that the regioselectivity of the reaction could be completely switched by changing the protection group of nitrogen, and a range of tetrahydropyrroles containing the monofluoromethyl group were efficiently synthesized [17]. Intermolecular aminofluorination of styrene was also achieved using a similar catalytic system [18]. In addition, our group also demonstrated that the intermolecular aminofluorination of styenes can be achieved using $N$-fluorobenze nesulfonimide (NFSI) as the source of nitrogen and fluorine in the presence of a palladium catalyst $[19,20]$. Since then, aminofluorination of alkenes has been extensively studied [21-24]. For example, Li et al. [25] reported silver-catalyzed radical aminofluorination of unactivated alkenes with Selectfluor as the fluorination reagent. Li et al. [26], Wang et al. [27], and

\footnotetext{
* Corresponding author. Tel: +86-21-54925346; Fax: +86-21- 64166128; E-mail: gliu@mail.sioc.ac.cn This work was supported by the National Basic Research Program of China (973 program, 2011CB808700), the National Natural Science Foundation of China (21225210, 20923005 and 21121062), the Science and Technology Commission of Shanghai Municipality (11JC1415000), and the CAS/SAFEA International Partnership Program for Creative Research Teams. DOI: 10.1016/S1872-2067(14)60164-9 | http://www.sciencedirect.com/science/journal/18722067 | Chin. J. Catal., Vol. 36, No. 1, January 2015
} 
Zhang et al. [28] separately reported intramolecular aminofluorination reactions of alkenes under metal-free conditions, and the related asymmetric aminofluorination reaction was described by Nevado et al. [29] and Toste et al. [30]. Because of the value of cyclic sulfamides in medicinal chemistry [31,32], we are interested in the construction of fluorinated heterocycles. We believe that palladium-catalyzed aminofluorination of $\mathrm{N}$-alkenylsulfamide might be an efficient way to synthesize those compounds. Herein, we report the results of our study on the intramolecular aminofluorination of allylic sulfamides.

\section{Experimental}

\subsection{General}

All commercially available compounds were used as received. ${ }^{1} \mathrm{H}$ and ${ }^{13} \mathrm{C}$ NMR spectra were recorded on an $\mathrm{Ag}$ ilent-400 MHz NMR spectrometer $\left(400 \mathrm{MHz}\right.$ for ${ }^{1} \mathrm{H} ; 376 \mathrm{MHz}$ for ${ }^{19} \mathrm{~F} ; 100 \mathrm{MHz}$ for ${ }^{13} \mathrm{C}$ ), and $\mathrm{CDCl}_{3}$ was purchased from $\mathrm{J} \& \mathrm{~K}$ (99.8 atom \% D, Shanghai, China). The chemical shifts $(\delta)$ are given in parts per million relative to the internal standards TMS ( 0 ppm for ${ }^{1} \mathrm{H}$ ) and $\mathrm{CDCl}_{3}\left(77.0\right.$ ppm for $\left.{ }^{13} \mathrm{C}\right)$. Flash column chromatography was performed on silica gel 60 (particle size 200-400 mesh ASTM, purchased from Yantai, China) and eluted with petroleum ether/ethyl acetate. All solvents were dried and purified according to the procedure in the "Purification of Laboratory Chemicals" [33]. AgF was purchased from Aldrich (>99.9\%, Shanghai, China). 1,1,1,3,3,3-hexafluoro-2-propanol (HFIP) was purchased from TCI (>99.0\%, Shanghai, China). Substrates 1a-1n were synthesized according to literature procedures [34].

\subsection{General procedure for aminofluorination of alkenes}

In a dry glass tube, $\mathrm{Pd}(\mathrm{OAc})_{2}(2.2 \mathrm{mg}, 0.01 \mathrm{mmol}), \mathrm{AgF}(76.2$ $\mathrm{mg}, 0.6 \mathrm{mmol}$ ), PhI(OPiv)2 (121.8 $\mathrm{mg}, 0.3 \mathrm{mmol}$ ), and alkene $(0.2 \mathrm{mmol})$ were dissolved in toluene $(1.0 \mathrm{~mL})$, and then HOAc $(120 \mu \mathrm{L}, 1 \mathrm{~mol} / \mathrm{L}$ in toluene, $0.12 \mathrm{mmol})$ was added. The reaction mixture was stirred at room temperature for $24 \mathrm{~h}$. The mixture was then filtered and the solid was washed with ethyl acetate. The combined filtrate was concentrated under vacuum. The residue was purified by column chromatography on silica gel with a gradient eluant of petroleum ether (or hexane) and ethyl acetate to give the products.

\subsection{Analytical data of the products}

2,6-Dibenzyl-4-fluoro-1,2,6-thiadiazinane 1,1-dioxide (2a). ${ }^{1} \mathrm{H}$ NMR (400 MHz, $\mathrm{CDCl}_{3}$ ): $\delta 7.30-7.41(\mathrm{~m}, 10 \mathrm{H}), 4.55(\mathrm{~d}, J=$ $13.8 \mathrm{~Hz}, 2 \mathrm{H}), 4.54(\mathrm{dt}, J=45.2,2.0 \mathrm{~Hz}, 1 \mathrm{H}), 4.41$ (d, $J=13.8 \mathrm{~Hz}$, 2H), 3.59 (dd, $J=40.8,15.2 \mathrm{~Hz}, 1 \mathrm{H}$ ), 3.43 (dd, $J=15.2,13.2 \mathrm{~Hz}$, $2 \mathrm{H}) ;{ }^{13} \mathrm{C}$ NMR $\left(100 \mathrm{MHz}, \mathrm{CDCl}_{3}\right): \delta 135.1,128.8,128.6,127.9$, 84.4 (d, $J=180.8 \mathrm{~Hz}$ ), 53.2 (d, $J=1.9 \mathrm{~Hz}$ ), 51.4 (d, $J=20.6 \mathrm{~Hz}$ ); ${ }^{19} \mathrm{~F}$ NMR (376 MHz, $\mathrm{CDCl}_{3}$ ): $\delta-180.9(\mathrm{dtt}, J=44.0,44.0,13.5$ $\mathrm{Hz}$ ); HRMS: $m / z$ (ESI) calcd. $[\mathrm{M}+\mathrm{H}]^{+}:$335.1230, found: 335.1239.

2-Benzyl-4-fluoro-6-(4-methylbenzyl)-1,2,6-thiadiazinane 1,1-dioxide (2b). ${ }^{1} \mathrm{H}$ NMR (400 MHz, $\mathrm{CDCl}_{3}$ ): $\delta$ 7.27-7.41 (m, $5 \mathrm{H}), 7.28(\mathrm{~d}, J=8.0 \mathrm{~Hz}, 2 \mathrm{H}), 7.18(\mathrm{~d}, J=7.6 \mathrm{~Hz}, 2 \mathrm{H}), 4.53(\mathrm{dt}, J=$ 45.2, $2.0 \mathrm{~Hz}, 1 \mathrm{H}), 4.48-4.55(\mathrm{~m}, 2 \mathrm{H}), 4.35-4.42(\mathrm{~m}, 2 \mathrm{H})$, 3.60-3.65 (m, 1H), 3.49-3.55 (m, 1H), 3.38-3.47 (m, 2H), 2.36 $(\mathrm{s}, 3 \mathrm{H}) ;{ }^{13} \mathrm{C} \mathrm{NMR}\left(100 \mathrm{MHz}, \mathrm{CDCl}_{3}\right): \delta 137.7,135.2,132.0,129.3$, 128.9, 128.8, 128.6, 127.9, 84.4 (d, $J=182.7 \mathrm{~Hz}$ ), 53.3 (d, $J=2.2$ $\mathrm{Hz}$ ), 53.1 (d, $J=2.1 \mathrm{~Hz}$ ), 51.4 (d, $J=20.6 \mathrm{~Hz}$ ), 51.2 (d, $J=20.6$ $\mathrm{Hz}$ ), 21.2; ${ }^{19} \mathrm{~F}$ NMR (376 MHz, $\mathrm{CDCl}_{3}$ ): $\delta-180.9$ (dtt, $J=41.4$, 41.4, $13.2 \mathrm{~Hz}$ ); HRMS: $m / z$ (ESI) calcd. $[\mathrm{M}+\mathrm{H}]^{+}: 349.1386$, found: 349.1367.

2-Benzyl-4-fluoro-6-(4-fluorobenzyl)-1,2,6-thiadiazinane 1,1-dioxide (2c). ${ }^{1} \mathrm{H}$ NMR (400 $\left.\mathrm{MHz}, \mathrm{CDCl}_{3}\right): \delta 7.31-7.40(\mathrm{~m}$, 7H), 7.04-7.08 (m, 2H), $4.56(\mathrm{dt}, J=45.2,2.0 \mathrm{~Hz}, 1 \mathrm{H}), 4.51(\mathrm{~d}, J=$ $13.6 \mathrm{~Hz}, 1 \mathrm{H}), 4.49(\mathrm{~d}, J=13.6 \mathrm{~Hz}, 1 \mathrm{H}), 4.38(\mathrm{~d}, J=13.6 \mathrm{~Hz}, 2 \mathrm{H})$, 3.64 (ddd, $J=15.2,5.6,2.0 \mathrm{~Hz}, 1 \mathrm{H}$ ), 3.54 (ddd, $J=15.2,5.6,2.0$ $\mathrm{Hz}, 1 \mathrm{H}), 3.37-3.47$ (m, 2H); ${ }^{13} \mathrm{C}$ NMR (100 MHz, $\left.\mathrm{CDCl}_{3}\right): \delta 162.5$ (d, $J=246.5 \mathrm{~Hz}$ ), 135.1, 130.9 (d, $J=3.2 \mathrm{~Hz}), 130.5$ (d, $J=8.2$ $\mathrm{Hz}), 128.8,128.7,128.0,115.6$ (d, $J=21.5 \mathrm{~Hz}$ ), 84.5 (d, $J=180.8$ $\mathrm{Hz}), 53.2(\mathrm{~d}, J=2.1 \mathrm{~Hz}), 52.7(\mathrm{~d}, J=2.3 \mathrm{~Hz}), 51.4(\mathrm{~d}, J=20.5 \mathrm{~Hz}$ ), $51.4(\mathrm{~d}, J=20.5 \mathrm{~Hz}) ;{ }^{19} \mathrm{~F}$ NMR (376 MHz, $\left.\mathrm{CDCl}_{3}\right): \delta-114.1(\mathrm{~m})$, -180.9 (dtt, $J=41.3,41.3,13.5 \mathrm{~Hz}$ ); HRMS: $m / z$ (ESI) calcd. [M $+\mathrm{H}]^{+}:$353.1135, found: 353.1145 .

2-Benzyl-6-butyl-4-fluoro-1,2,6-thiadiazinane 1,1-dioxide (2d). ${ }^{1} \mathrm{H}$ NMR (400 MHz, $\left.\mathrm{CDCl}_{3}\right): \delta 7.25-7.36(\mathrm{~m}, 5 \mathrm{H}), 4.58(\mathrm{dt}, J$ $=45.2,2.4 \mathrm{~Hz}, 1 \mathrm{H}), 4.42(\mathrm{~d}, J=13.6 \mathrm{~Hz}, 1 \mathrm{H}), 4.34(\mathrm{~d}, J=13.6 \mathrm{~Hz}$, 1H), 3.74 (ddd, $J=40.0,14.8,2.0 \mathrm{~Hz}, 1 \mathrm{H}$ ), 3.49-3.64 (m, 2H), 3.23-3.42 (m, 3H), $1.60(\mathrm{~m}, 2 \mathrm{H}), 1.40(\mathrm{~m}, 2 \mathrm{H}), 0.96(\mathrm{t}, J=7.2 \mathrm{~Hz}$, $3 \mathrm{H})$; ${ }^{13} \mathrm{C}$ NMR $\left(100 \mathrm{MHz}, \mathrm{CDCl}_{3}\right): \delta 135.2,128.8,128.5,127.8$, $84.3(\mathrm{~d}, J=180.9 \mathrm{~Hz}), 53.3(\mathrm{~d}, J=2.4 \mathrm{~Hz}), 52.8$ (d, $J=20.6 \mathrm{~Hz}$ ), 51.1 (d, $J=20.4 \mathrm{~Hz}), 49.8,29.9,19.6,13.6$; ${ }^{19} \mathrm{~F}$ NMR $(376 \mathrm{MHz}$, $\mathrm{CDCl}_{3}$ ): $\delta-181.6(\mathrm{dtt}, J=41.4,41.4,13.5 \mathrm{~Hz})$; HRMS: $m / z(\mathrm{ESI})$ calcd. $[\mathrm{M}+\mathrm{H}]^{+}:$301.1386, found: 301.1379 .

2-Benzyl-4-fluoro-6-neopentyl-1,2,6-thiadiazinane 1,1-dioxide (2f). ${ }^{1} \mathrm{H}$ NMR (400 MHz, $\mathrm{CDCl}_{3}$ ): $\delta 7.25-7.36(\mathrm{~m}, 5 \mathrm{H}), 4.55$ (d, $J=45.2 \mathrm{~Hz}, 1 \mathrm{H}), 4.46$ (d, $J=14.0 \mathrm{~Hz}, 1 \mathrm{H}), 4.34$ (d, $J=14.0 \mathrm{~Hz}$, 1H), 3.92 (dd, $J=40.0,14.8 \mathrm{~Hz}, 1 \mathrm{H}), 3.62-3.70(\mathrm{~m}, 1 \mathrm{H}), 3.53$ (dd, $J=40.8,14.8 \mathrm{~Hz}, 1 \mathrm{H}$ ), 3.34-3.42 (m, 1H), 3.19 (d, $J=14.4$ $\mathrm{Hz}, 1 \mathrm{H}$ ), 2.99 (d, $J=14.8 \mathrm{~Hz}, 1 \mathrm{H}), 0.98$ (d, $J=1.2 \mathrm{~Hz}, 9 \mathrm{H}) ;{ }^{13} \mathrm{C}$ NMR (100 MHz, $\left.\mathrm{CDCl}_{3}\right): \delta 135.2,128.8,128.6,127.9,84.5(\mathrm{~d}, J=$ $180.5 \mathrm{~Hz}), 61.4(\mathrm{~d}, J=1.6 \mathrm{~Hz}), 56.2(\mathrm{~d}, J=19.9 \mathrm{~Hz}), 53.3(\mathrm{~d}, J=$ $2.2 \mathrm{~Hz}$ ), 51.3 (d, $J=20.5 \mathrm{~Hz}), 33.2,27.3 ;{ }^{19} \mathrm{~F}$ NMR (376 MHz, $\left.\mathrm{CDCl}_{3}\right): \delta-182.6(\mathrm{dtt}, J=41.4,41.4,12.5 \mathrm{~Hz})$; HRMS: $m / z$ (ESI) calcd. $[\mathrm{M}+\mathrm{H}]^{+}:$315.1543, found: 315.1551 .

2-Benzyl-4-fluoro-6-methyl-1,2,6-thiadiazinane 1,1-dioxide (2g). ${ }^{1} \mathrm{H}$ NMR (400 MHz, CDCl 3$): \delta 7.29-7.39(\mathrm{~m}, 5 \mathrm{H}), 4.60(\mathrm{dt}, J$ $=45.2,2.0 \mathrm{~Hz}, 1 \mathrm{H}), 4.47(\mathrm{~d}, J=13.6 \mathrm{~Hz}, 1 \mathrm{H}), 4.33(\mathrm{~d}, J=14.0 \mathrm{~Hz}$, 1H), 3.79 (ddd, $J=41.2,15.2,2.4 \mathrm{~Hz}, 1 \mathrm{H}), 3.36-3.63(\mathrm{~m}, 3 \mathrm{H})$, $2.97(\mathrm{~d}, J=1.2 \mathrm{~Hz}, 3 \mathrm{H}) ;{ }^{13} \mathrm{C}$ NMR (100 MHz, $\left.\mathrm{CDCl}_{3}\right): \delta 135.2$, 128.8, 128.6, 127.9, 84.4 (d, $J=181.0 \mathrm{~Hz}$ ), 56.1 (d, $J=20.6 \mathrm{~Hz}$ ), $53.1(\mathrm{~d}, J=2.1 \mathrm{~Hz}), 51.4(\mathrm{~d}, J=20.6 \mathrm{~Hz}), 38.5(\mathrm{~d}, J=1.9 \mathrm{~Hz}) ;{ }^{19} \mathrm{~F}$ NMR (376 MHz, $\left.\mathrm{CDCl}_{3}\right): \delta-180.6(\mathrm{dtt}, J=42.1,42.1,12.8 \mathrm{~Hz}$ ); HRMS: $m / z$ (ESI) calcd. [M + H]+: 259.0917, found: 259.0913 .

2-Benzyl-4-fluoro-6-phenyl-1,2,6-thiadiazinane 1,1-dioxide (2h). ${ }^{1} \mathrm{H}$ NMR (400 MHz, $\mathrm{CDCl}_{3}$ ): $\delta 7.28-7.45$ (m, 10H), 4.70 (dt, $J=44.8,2.0 \mathrm{~Hz}, 1 \mathrm{H}), 4.76(\mathrm{~d}, J=13.6 \mathrm{~Hz}, 1 \mathrm{H}), 4.42$ (dd, $J=13.6$, $1.6 \mathrm{~Hz}, 1 \mathrm{H}$ ), 4.22 (ddd, $J=39.6,14.0,2.0 \mathrm{~Hz}, 1 \mathrm{H}$ ), 3.76-3.91 (m, $2 \mathrm{H}), 3.48-3.56(\mathrm{~m}, 1 \mathrm{H}) ;{ }^{13} \mathrm{C} \mathrm{NMR}\left(100 \mathrm{MHz}, \mathrm{CDCl}_{3}\right): \delta 141.1$, 
135.1, 129.2, 129.1, 128.6, 128.0, 127.5, 126.5 (d, $J=0.7 \mathrm{~Hz}$ ), $84.3(\mathrm{~d}, J=181.9 \mathrm{~Hz}), 56.9$ (d, $J=21.7 \mathrm{~Hz}), 54.4$ (d, $J=3.9 \mathrm{~Hz}$ ), 50.4 (d, $J=19.8 \mathrm{~Hz}) ;{ }^{19} \mathrm{~F}$ NMR (376 MHz, $\left.\mathrm{CDCl}_{3}\right): \delta-180.9$ (dtt, $J$ = 42.8, 42.8, $13.2 \mathrm{~Hz}$ ); HRMS: $m / z$ (ESI) calcd. $[\mathrm{M}+\mathrm{H}]^{+}$: 321.1073, found: 321.1068 .

tert-Butyl 2-(6-benzyl-4-fluoro-1,1-dioxido-1,2,6-thiadiazinan-2-yl)acetate (2i). ${ }^{1} \mathrm{H}$ NMR $\left(400 \mathrm{MHz} \mathrm{CDCl}_{3}\right): \delta$ 7.29-7.38 (m, 5H), $4.61(\mathrm{dt}, J=44.8,2.4 \mathrm{~Hz}, 1 \mathrm{H}), 4.48(\mathrm{~d}, J=14.0 \mathrm{~Hz}, 1 \mathrm{H})$, 4.32 (d, $J=13.6 \mathrm{~Hz}, 1 \mathrm{H}), 4.05(\mathrm{~d}, J=17.2 \mathrm{~Hz}, 1 \mathrm{H}), 3.97$ (d, $J=$ $17.6 \mathrm{~Hz}, 1 \mathrm{H}), 3.88-3.99$ (m, 1H), 3.71-3.79 (m, 1H), 3.52-3.66 (m, 1H), 3.37-3.46 (m, 1H), $1.50(\mathrm{~s}, 9 \mathrm{H}) ;{ }^{13} \mathrm{C}$ NMR $(100 \mathrm{MHz}$, $\left.\mathrm{CDCl}_{3}\right): \delta 167.4,134.9,128.8,128.6,127.9,84.3(\mathrm{~d}, J=180.5$ $\mathrm{Hz}), 82.3,53.8$ (d, $J=20.5 \mathrm{~Hz}), 53.2$ (d, $J=2.2 \mathrm{~Hz}$ ), 51.5, 51.21, 27.9; ${ }^{19} \mathrm{~F}$ NMR (376 MHz, $\mathrm{CDCl}_{3}$ ): $\delta-181.2$ (dtt, $J=41.0,41.0$, $13.5 \mathrm{~Hz}$ ); HRMS: $m / z$ (ESI) calcd. $[\mathrm{M}+\mathrm{H}]^{+}:$359.1441, found: 359.1433 .

2,6-Dibenzyl-4-fluoro-4-methyl-1,2,6-thiadiazinane 1,1-dioxide (2j). ${ }^{1} \mathrm{H}$ NMR (400 MHz, $\left.\mathrm{CDCl}_{3}\right): \delta 7.31-7.38(\mathrm{~m}, 10 \mathrm{H})$, $4.57(\mathrm{~d}, J=13.6 \mathrm{~Hz}, 2 \mathrm{H}), 4.42(\mathrm{~d}, J=13.6 \mathrm{~Hz}, 2 \mathrm{H}), 3.48$ (dd, $J=$ $38.4,14.8 \mathrm{~Hz}, 2 \mathrm{H}), 3.23$ (dd, $J=13.6,12.0 \mathrm{~Hz}, 2 \mathrm{H}), 1.16(\mathrm{~d}, J=$ $20.4 \mathrm{~Hz}, 3 \mathrm{H}) ;{ }^{13} \mathrm{C}$ NMR $\left(100 \mathrm{MHz}, \mathrm{CDCl}_{3}\right): \delta 135.5,128.7,128.6$, 127.8, 90.2 (d, $J=177.2 \mathrm{~Hz}$ ), 55.7 (d, $J=21.3 \mathrm{~Hz}$ ), 53.0 (d, $J=2.6$ $\mathrm{Hz}), 21.8(\mathrm{~d}, J=22.4 \mathrm{~Hz}) ;{ }^{19} \mathrm{~F}$ NMR (376 MHz, $\left.\mathrm{CDCl}_{3}\right): \delta-148.6$ (m); HRMS: $m / z$ (ESI) calcd. $[\mathrm{M}+\mathrm{H}]^{+}:$349.1386, found: 349.1380 .

2,6-Dibenzyl-4-fluoro-3-methyl-1,2,6-thiadiazinane 1,1-dioxide (2k). ${ }^{1} \mathrm{H}$ NMR (400 MHz, $\left.\mathrm{CDCl}_{3}\right): \delta 7.29-7.42(\mathrm{~m}, 10 \mathrm{H})$, 4.59 (d, $J=14.8 \mathrm{~Hz}, 1 \mathrm{H}), 4.54(\mathrm{~d}, J=14.4 \mathrm{~Hz}, 1 \mathrm{H}), 4.50$ (d, $J=$ $14.0 \mathrm{~Hz}, 1 \mathrm{H}$ ), 4.42 (d, $J=14.0 \mathrm{~Hz}, 1 \mathrm{H}$ ), 4.35 (ddd, $J=44.8,5.2$, $3.2 \mathrm{~Hz}, 1 \mathrm{H}$ ), 3.75-3.82 (m, 1H), 3.66 (ddd, $J=37.2,14.8,2.0 \mathrm{~Hz}$, $1 \mathrm{H}), 3.33-3.41(\mathrm{~m}, 1 \mathrm{H}), 1.34(\mathrm{dd}, J=7.6,0.8 \mathrm{~Hz}, 1 \mathrm{H}) ;{ }^{13} \mathrm{C} \mathrm{NMR}$ $\left(100 \mathrm{MHz}, \mathrm{CDCl}_{3}\right): \delta 136.2,135.2,128.9,128.6,128.3,127.9$, 127.8, 87.67 (d, $J=180.7 \mathrm{~Hz}), 57.99$ (d, $J=21.1 \mathrm{~Hz}), 53.14$ (d, $J=$ $2.4 \mathrm{~Hz}$ ), 52.46 (d, $J=1.7 \mathrm{~Hz}), 48.13$ (d, $J=21.5 \mathrm{~Hz}), 15.58$ (d, $J=$ $9.1 \mathrm{~Hz}$ ); ${ }^{19} \mathrm{~F}$ NMR (376 MHz, $\mathrm{CDCl}_{3}$ ): $\delta-176.9$ (m). HRMS: $m / z$ (ESI) calcd. $[\mathrm{M}+\mathrm{H}]^{+}:$349.1386, found: 349.1379 .

6-Benzyl-4-fluoro-2-neopentyl-3-phenyl-1,2,6-thiadiazinan e 1,1-dioxide (2l). ${ }^{1} \mathrm{H}$ NMR (400 MHz, $\mathrm{CDCl}_{3}$ ): $\delta 7.55$ (d, $J=7.6$ $\mathrm{Hz}, 2 \mathrm{H}), 7.30-7.40(\mathrm{~m}, 8 \mathrm{H}), 5.11-5.14(\mathrm{~m}, 1 \mathrm{H}), 5.07$ (d, $J=38.0$ $\mathrm{Hz}, 1 \mathrm{H}), 4.40$ (d, $J=13.6 \mathrm{~Hz}, 1 \mathrm{H}), 4.39$ (d, $J=13.6 \mathrm{~Hz}, 1 \mathrm{H}$ ), $3.47-3.58$ (ddd, $J=30.0,14.0,2.4 \mathrm{~Hz}, 1 \mathrm{H}$ ), 3.35-3.42 (m, $1 \mathrm{H}$ ), 3.34 (d, $J=14.8 \mathrm{~Hz}, 1 \mathrm{H}$ ), 3.22 (d, $J=14.8 \mathrm{~Hz}, 1 \mathrm{H}), 0.86$ (s, 9H); ${ }^{13} \mathrm{C}$ NMR (100 MHz, $\left.\mathrm{CDCl}_{3}\right): \delta 135.1,134.70(\mathrm{~d}, J=7.9 \mathrm{~Hz})$, 128.8, 128.6, 128.4, 128.2, 128.2, 128.0, 86.3 (d, $J=179.9 \mathrm{~Hz}$ ), 69.6 (d, $J=22.6 \mathrm{~Hz}), 61.4,52.9$ (d, $J=1.9 \mathrm{~Hz}), 48.8$ (d, $J=23.5$ $\mathrm{Hz}), 33.1,27.7 ;{ }^{19} \mathrm{~F}$ NMR (376 $\left.\mathrm{MHz}, \mathrm{CDCl}_{3}\right): \delta-180.5(\mathrm{~m})$; HRMS: $m / z$ (ESI) calcd. [M + H]+: 391.1856, found: 391.1847 .

2,6-Dibenzyl-4-fluoro-3-phenyl-1,2,6-thiadiazinane 1,1-dioxide (2m). ${ }^{1} \mathrm{H}$ NMR (400 MHz, $\left.\mathrm{CDCl}_{3}\right): \delta 7.33-7.42(\mathrm{~m}, 5 \mathrm{H})$, 7.17-7.28 (m, 8H), 7.02-7.05 (m, 2H), 4.91 (ddt, $J=45.2,8.4$, $4.8 \mathrm{~Hz}, 1 \mathrm{H}), 4.76(\mathrm{dd}, J=14.8,10.4 \mathrm{~Hz}, 1 \mathrm{H}), 4.50(\mathrm{~d}, J=15.2 \mathrm{~Hz}$, $1 \mathrm{H}), 4.46$ (d, $J=15.2 \mathrm{~Hz}, 1 \mathrm{H}), 4.31(\mathrm{~d}, J=15.2 \mathrm{~Hz}, 1 \mathrm{H}), 4.26(\mathrm{~d}, J$ $=15.2 \mathrm{~Hz}, 1 \mathrm{H}), 3.60-3.67(\mathrm{~m}, 1 \mathrm{H}), 3.48-3.55(\mathrm{~m}, 1 \mathrm{H}) ;{ }^{13} \mathrm{C} \mathrm{NMR}$ (100 MHz, $\left.\mathrm{CDCl}_{3}\right): \delta 136.4,134.9,134.40(\mathrm{~d}, J=2.5 \mathrm{~Hz}), 128.87$, $128.79,128.77,128.71,128.5,128.3,128.0,127.4,84.9(\mathrm{~d}, J=$ $181.1 \mathrm{~Hz}), 66.6$ (d, $J=25.5 \mathrm{~Hz}), 53.3,50.7,48.8(\mathrm{~d}, J=28.2 \mathrm{~Hz}$ ); ${ }^{19} \mathrm{~F}$ NMR (376 MHz, $\mathrm{CDCl}_{3}$ ): $\delta-180.5(\mathrm{ddt}, J=48.4,11.2,11.2$
Hz); HRMS: $m / z$ (ESI) calcd. $[\mathrm{M}+\mathrm{H}]^{+}:$411.1543, found: 411.1537.

\subsection{Analytical data for the substrates}

$N$-allylic- $N, N$-dibenzylsulfamide (1a). ${ }^{1} \mathrm{H}$ NMR $(400 \mathrm{MHz}$, $\left.\mathrm{CDCl}_{3}\right): \delta 7.23-7.34(\mathrm{~m}, 10 \mathrm{H}), 5.73-5.83(\mathrm{~m}, 1 \mathrm{H}), 5.13-5.22(\mathrm{~m}$, 2H), $4.6(\mathrm{br}, 1 \mathrm{H}), 4.37(\mathrm{~s}, 2 \mathrm{H}), 4.15(\mathrm{~d}, J=6.0 \mathrm{~Hz}, 2 \mathrm{H}), 3.72(\mathrm{~d}, J=$ $6.4 \mathrm{~Hz}, 2 \mathrm{H}) ;{ }^{13} \mathrm{C}$ NMR $\left(100 \mathrm{MHz}, \mathrm{CDCl}_{3}\right): \delta 136.7,136.3,132.7$, 128.7, 128.6, 128.4, 127.9, 127.8, 127.7, 119.5, 50.5, 49.8, 47.2; HRMS: $m / z$ (ESI) calcd. [M+H]+: 317.1324, found: 317.1330.

$N$-allylic- $N$-benzyl- $N$ - $\left(p\right.$-tolylmethyl)sulfamide $\quad(\mathbf{1 b}) . \quad{ }^{1} \mathrm{H}$ NMR (400 MHz, $\mathrm{CDCl}_{3}$ ): $\delta$ 7.26-7.34 (m, 5H), 7.10-7.17 (m, 4H), 5.74-5.84 (m, 1H), 5.12-5.22 (m, 2H), $4.60(\mathrm{br}, 1 \mathrm{H}), 4.35(\mathrm{~s}$, $2 \mathrm{H}), 4.11(\mathrm{~d}, J=6.0 \mathrm{~Hz}, 2 \mathrm{H}), 3.71(\mathrm{~d}, J=6.4 \mathrm{~Hz}, 2 \mathrm{H}), 2.31(\mathrm{~s}, 3 \mathrm{H})$; ${ }^{13} \mathrm{C}$ NMR $\left(100 \mathrm{MHz} \mathrm{CDCl}_{3}\right): \delta 137.5,136.2,133.6,132.7,129.3$, 128.52, 128.49, 127.8, 127.7, 119.4, 50.3, 49.6, 46.9, 21.0; HRMS: $m / z$ (ESI) calcd. [M + H]+: 331.1480, found: 331.1491 .

$N$-allylic- $N$-benzyl- $N$-( $p$-fluorophenyl)methylsulfamide (1c). ${ }^{1} \mathrm{H}$ NMR (400 MHz, $\mathrm{CDCl}_{3}$ ): $\delta 7.27-7.36(\mathrm{~m}, 5 \mathrm{H}), 7.21-7.27$ (m, 2H), 6.97-7.03 (m, 2H), 5.75-5.85 (m, 1H), 5.13-5.24 (m, 2H), $4.59(\mathrm{br}, 1 \mathrm{H}), 4.36(\mathrm{~s}, 2 \mathrm{H}), 4.11(\mathrm{~d}, J=6.0 \mathrm{~Hz}, 2 \mathrm{H}), 3.73(\mathrm{~d}, J$ $=6.4 \mathrm{~Hz}, 2 \mathrm{H}) ;{ }^{13} \mathrm{C}$ NMR $\left(100 \mathrm{MHz}, \mathrm{CDCl}_{3}\right): \delta 162.3(\mathrm{~d}, J=245.2$ $\mathrm{Hz}), 136.1,132.6,132.5(\mathrm{~d}, J=3.1 \mathrm{~Hz}), 129.6(\mathrm{~d}, J=8.0 \mathrm{~Hz})$, 128.6, 128.5, 127.8, 119.6, 115.5 (d, $J=21.7 \mathrm{~Hz}$ ), 50.4, 49.8, 46.5; ${ }^{19} \mathrm{~F}$ NMR (376M, $\mathrm{CDCl}_{3}$ ): $\delta-114.25$ (m); HRMS: $m / z$ (ESI) calcd. $[\mathrm{M}+\mathrm{H}]^{+}:$335.1230, found: 335.1240 .

$N$-allylic- $N$-benzyl- $N$-butylsulfamide (1d). ${ }^{1} \mathrm{H}$ NMR $(400$ $\mathrm{MHz}, \mathrm{CDCl}_{3}$ ): $\delta 7.25-7.34(\mathrm{~m}, 5 \mathrm{H}), 5.78-5.88(\mathrm{~m}, 1 \mathrm{H}), 5.12-5.22$ $(\mathrm{m}, 2 \mathrm{H}), 4.74(\mathrm{br}, 1 \mathrm{H}), 4.36(\mathrm{~s}, 2 \mathrm{H}), 3.71(\mathrm{~d}, J=6.4 \mathrm{~Hz}, 2 \mathrm{H})$, 2.95-3.00 (m, 2H), 1.44-1.51 (m, 2H), 1.28-1.37 (m, 2H), 0.90 $(\mathrm{t}, J=7.6 \mathrm{~Hz}, 3 \mathrm{H}) ;{ }^{13} \mathrm{C}$ NMR $\left(100 \mathrm{MHz}, \mathrm{CDCl}_{3}\right): \delta 136.3,132.7$, 128.4, 127.5, 119.1, 50.2, 49.5, 42.7, 31.4, 19.7, 13.5; HRMS: $m / z$ (ESI) calcd. [M + H]+: 283.1480, found: 283.1485.

$N$-allylic- $N$-benzyl- $N$-phenylsulfamide (1e). ${ }^{1} \mathrm{H}$ NMR $(400$ $\left.\mathrm{MHz}, \mathrm{CDCl}_{3}\right): \delta 7.23-7.28(\mathrm{~m}, 2 \mathrm{H}), 7.16-7.19(\mathrm{~m}, 5 \mathrm{H}), 7.06-7.10$ $(\mathrm{m}, 3 \mathrm{H}), 5.46-5.56(\mathrm{~m}, 1 \mathrm{H}), 4.96-5.06(\mathrm{~m}, 2 \mathrm{H}), 4.34(\mathrm{~s}, 2 \mathrm{H})$, 3.69 (d, $J=6.8 \mathrm{~Hz}, 2 \mathrm{H}) ;{ }^{13} \mathrm{C}$ NMR $\left(100 \mathrm{MHz}, \mathrm{CDCl}_{3}\right): \delta 137.4$, 135.7, 132.3, 129.4, 128.62, 128.61, 127.8, 124.5, 120.2, 119.7, 50.6, 49.8; HRMS: $m / z$ (ESI) calcd. $[\mathrm{M}+\mathrm{H}]^{+}:$303.1167, found: 303.1177.

$N$-allylic- $N$-neopentyl- $N$-benzylsulfamide (1f). ${ }^{1} \mathrm{H}$ NMR $(400$ $\left.\mathrm{MHz}, \mathrm{CDCl}_{3}\right): \delta 7.25-7.36(\mathrm{~m}, 5 \mathrm{H}), 5.88-5.99(\mathrm{~m}, 1 \mathrm{H}), 5.21-5.25$ $(\mathrm{m}, 2 \mathrm{H}), 4.45(\mathrm{br}, 1 \mathrm{H}), 4.20$ (d, $J=6.0 \mathrm{~Hz}, 2 \mathrm{H}), 3.89(\mathrm{~d}, J=6.8 \mathrm{~Hz}$, 2H). 3.03 (s, 2H), $\left.0.96(\mathrm{~s}, 9 \mathrm{H}) ;{ }^{13} \mathrm{C} \mathrm{NMR} \mathrm{(100} \mathrm{MHz,} \mathrm{CDCl}_{3}\right): \delta$ 136.8, 133.4, 128.7, 127.9, 127.9, 119.4, 58.5, 53.3, 47.5, 33.5, 28.2; HRMS: $m / z$ (ESI) calcd. $[\mathrm{M}+\mathrm{H}]^{+}:$297.1637, found: 297.1630.

$N$-allylic- $N$-methyl- $N$-benzylsulfamide (1g). ${ }^{1} \mathrm{H}$ NMR $(400$ $\mathrm{MHz}, \mathrm{CDCl}_{3}$ ): $\delta$ 7.25-7.35 (m, 5H), 5.69-5.79 (m, 1H), 5.18-5.24 $(\mathrm{m}, 2 \mathrm{H}), 4.89(\mathrm{br}, 1 \mathrm{H}), 4.16(\mathrm{~d}, J=6.0 \mathrm{~Hz}, 2 \mathrm{H}), 3.67$ (d, $J=6.4 \mathrm{~Hz}$, 2H). 2.69 (s, 3H); ${ }^{13} \mathrm{C}$ NMR (100 MHz, $\left.\mathrm{CDCl}_{3}\right): \delta 136.9,132.8$, 128.6, 127.8, 127.7, 118.7, 53.0, 47.2, 34.2; HRMS: $m / z$ (ESI) calcd. [M+ H]+: 241.1011, found: 241.1011.

$N$-allylic- $N$-phenyl- $N$-benzylsulfamide (1h). ${ }^{1} \mathrm{H}$ NMR $(400$ $\left.\mathrm{MHz}, \mathrm{CDCl}_{3}\right): \delta$ 7.23-7.33 (m, 10H), 5.74-5.84 (m, 1H), 5.06-5.11 (m, 2H), 4.69 (br, 1H), 4.18-4.21 (m, 4H); ${ }^{13} \mathrm{C}$ NMR 
(100 MHz, $\left.\mathrm{CDCl}_{3}\right): \delta 140.2,136.6,133.2,129.2,128.7,128.3$, 127.9, 127.8, 127.6, 118.7, 54.6, 47.5; HRMS: $m / z$ (ESI) calcd. $[\mathrm{M}+\mathrm{H}]+$ : 303.1167, found: 303.1168.

tert-Butyl $N$-allylic-(benzylaminosulfonyl)aminoacetate (1i). ${ }^{1} \mathrm{H}$ NMR $\left(400 \mathrm{MHz}, \mathrm{CDCl}_{3}\right): \delta 7.26-7.38(\mathrm{~m}, 5 \mathrm{H}), 5.76-5.86(\mathrm{~m}$, $1 \mathrm{H}), 5.22-5.27(\mathrm{~m}, 2 \mathrm{H}), 5.11(\mathrm{br}, 1 \mathrm{H}), 4.33(\mathrm{~d}, J=6.0 \mathrm{~Hz}, 2 \mathrm{H})$, $3.91(\mathrm{~s}, 2 \mathrm{H}), 3.88$ (d, J = 6.4 Hz, 2H), $1.45(\mathrm{~s}, 9 \mathrm{H}) ;{ }^{13} \mathrm{C}$ NMR $(100$ $\left.\mathrm{MHz}, \mathrm{CDCl}_{3}\right): \delta 169.6,137.3,132.7,128.6,127.9,127.7,119.5$, 82.5, 51.3, 49.1, 47.1, 27.9; HRMS: $m / z$ (ESI) calcd. [M + Na]+: 363.1354, measured: 363.1364 .

$N$-(2-methylprop-2-en-1)- $N, N^{\prime}$-dibenzylsulfamide $\quad(\mathbf{1 j}) .{ }^{1} \mathrm{H}$ NMR (400 MHz, $\left.\mathrm{CDCl}_{3}\right): \delta 7.21-7.36(\mathrm{~m}, 10 \mathrm{H}), 4.97(\mathrm{~s}, 1 \mathrm{H}), 4.92$ $(\mathrm{s}, 1 \mathrm{H}), 4.37(\mathrm{~s}, 2 \mathrm{H}), 4.27(\mathrm{t}, J=5.6 \mathrm{~Hz}, 1 \mathrm{H}), 4.10(\mathrm{~d}, J=6.0 \mathrm{~Hz}$, $2 \mathrm{H}), 3.74(\mathrm{~s}, 2 \mathrm{H}), 1.73(\mathrm{~s}, 3 \mathrm{H}) ;{ }^{13} \mathrm{C}$ NMR $\left(100 \mathrm{MHz}, \mathrm{CDCl}_{3}\right): \delta$ 140.2, 136.6, 136.2, 128.9, 128.7, 128.6, 127.9, 127.8, 114.7, 53.5, 50.6, 47.3, 20.1; HRMS: $\mathrm{m} / \mathrm{z}$ (ESI) calcd. $[\mathrm{M}+\mathrm{H}]^{+}$: 331.1480, found: 331.1478 .

$N$-(1-methylprop-2-en-1)- $N, N^{\prime}$-dibenzylsulfamide $(\mathbf{1} \mathbf{k}) .{ }^{1} \mathrm{H}$ $\operatorname{NMR}\left(400 \mathrm{MHz}, \mathrm{CDCl}_{3}\right): \delta 7.38(\mathrm{~d}, J=7.2 \mathrm{~Hz}, 2 \mathrm{H}), 7.22-7.32(\mathrm{~m}$, $6 \mathrm{H}), 7.16(\mathrm{~d}, J=6.4 \mathrm{~Hz}, 2 \mathrm{H}), 5.93-6.01(\mathrm{~m}, 1 \mathrm{H}), 5.17-5.23(\mathrm{~m}$, $2 \mathrm{H}), 4.50(\mathrm{~m}, 1 \mathrm{H}), 4.35(\mathrm{~d}, J=16.0 \mathrm{~Hz}, 1 \mathrm{H}), 4.19-4.23(\mathrm{~m}, 2 \mathrm{H})$, 3.94-4.03 (m, 2H), $1.28(\mathrm{~d}, J=6.8 \mathrm{~Hz}, 3 \mathrm{H}) ;{ }^{13} \mathrm{C}$ NMR $(100 \mathrm{MHz}$, $\left.\mathrm{CDCl}_{3}\right): \delta 138.3,138.2,136.4,128.5,128.4,128.2,127.8,127.7$, 127.4, 117.0, 56.2, 47.9, 47.1, 17.6; HRMS: $m / z$ (ESI) calcd. [M + $\mathrm{H}]+:$ 331.1480, found: 331.1469.

$N$-(2-phenylprop-2-en-1)- $N$-neopentyl- $N$-benzylsulfamide (11). ${ }^{1} \mathrm{H}$ NMR $\left(400 \mathrm{MHz}, \mathrm{CDCl}_{3}\right): \delta 7.54(\mathrm{~d}, J=7.6 \mathrm{~Hz}, 2 \mathrm{H})$, 7.23-7.33 (m, 6H), 7.12-7.14 (m, 2H), 6.51-6.60 (m, 1H), 5.43 (dd, $J=10.0 \mathrm{~Hz}, J=0.8 \mathrm{~Hz}, 1 \mathrm{H}), 5.30(\mathrm{~d}, J=17.2 \mathrm{~Hz}, 1 \mathrm{H}), 4.97(\mathrm{~d}$, $J=8.4 \mathrm{~Hz}, 1 \mathrm{H}), 4.11(\mathrm{dd}, J=13.6,7.2 \mathrm{~Hz}, 1 \mathrm{H}), 3.92(\mathrm{dd}, J=13.2$ $\mathrm{Hz}, J=5.2 \mathrm{~Hz}, 1 \mathrm{H}), 3.51(\mathrm{br}, 1 \mathrm{H}), 3.26(\mathrm{~d}, J=15.2 \mathrm{~Hz}, 1 \mathrm{H}), 3.12$ (d, $J=14.8 \mathrm{~Hz}, 1 \mathrm{H}), 1.00$ (s, 9H); ${ }^{13} \mathrm{C}$ NMR $\left(100 \mathrm{MHz}, \mathrm{CDCl}_{3}\right): \delta$ 139.4, 136.7, 135.9, 128.5, 128.4, 128.3, 127.9, 127.8, 127.7, 119.6, 68.6, 60.8, 47.6, 33.3, 28.5; HRMS: $m / z$ (ESI) calcd. [M + $\mathrm{H}]^{+}:$373.1950, found: 373.1933 .

$N$-(2-phenylprop-2-en-1)- $N, N^{\prime}$-dibenzylsulfamide $(\mathbf{1 m}) .{ }^{1} \mathrm{H}$ NMR (400 MHz, $\left.\mathrm{CDCl}_{3}\right): \delta$ 7.06-7.43 (m, 15H), 6.21-6.30 (m, $1 \mathrm{H}), 5.57(\mathrm{~d}, J=6.8 \mathrm{~Hz}, 1 \mathrm{H}), 5.41(\mathrm{~d}, J=10.0 \mathrm{~Hz}, 1 \mathrm{H}), 5.38(\mathrm{~d}, J=$ $16.8 \mathrm{~Hz}, 1 \mathrm{H}), 4.38(\mathrm{~d}, J=15.2 \mathrm{~Hz}, 1 \mathrm{H}), 4.28(\mathrm{~d}, J=15.2 \mathrm{~Hz}, 1 \mathrm{H})$, 3.94-3.99 (m, 1H), 3.82-3.84 (m, 2H); ${ }^{13} \mathrm{C}$ NMR $(100 \mathrm{MHz}$, $\left.\mathrm{CDCl}_{3}\right): \delta 138.7,137.4,136.3,134.9,128.7,128.6,128.55$, 128.50, 128.45, 128.03, 127.9, 127.7, 127.6, 119.5, 65.1, 49.5, 47.1; HRMS: $m / z$ (ESI) calcd. $[\mathrm{M}+\mathrm{H}]^{+}$: 393.1637, found: 393.1617.

$N$-((E)-2-methylbut-2-en-1)-N,N'-dibenzylsulfamide (1n). ${ }^{1} \mathrm{H}$ NMR $\left(400 \mathrm{MHz}, \mathrm{CDCl}_{3}\right): \delta 7.20-7.33(\mathrm{~m}, 10 \mathrm{H}), 5.35(\mathrm{q}, J=5.2$ $\mathrm{Hz}, 1 \mathrm{H}), 4.47$ (br, 1H), 4.28 (s, 2H), 4.07 (d, J = 6.4 Hz, 2H), 3.69 (s, $2 \mathrm{H}), 1.58(\mathrm{~d}, J=5.6 \mathrm{~Hz}, 3 \mathrm{H}), 1.57(\mathrm{~s}, 3 \mathrm{H}) ;{ }^{13} \mathrm{C}$ NMR $(100 \mathrm{MHz}$, $\mathrm{CDCl}_{3}$ ): $\delta 136.6,136.5,130.6,128.7,128.5,128.3,127.8,127.7$, 127.5, 124.5, 55.7, 50.3, 47.1, 13.7, 13.3; HRMS: $m / z$ (ESI) calcd. $[\mathrm{M}+\mathrm{H}]+:$ 345.1637, found: 345.1639 .

\section{Results and discussion}

The initial study focused on the reaction of $N$-alkenylsulfamide 1a. When the reaction was carried out under our previous conditions [17], the aminofluorinated product 2a was obtained in $45 \%$ yield with high regioselectivity (Table 1, entry 1). In our previous study, addition of acidic additives, such as $\left(\mathrm{CF}_{3}\right)_{2} \mathrm{CHOH}$ (HFIP), was helpful to improve aminofluorination with good mass balance [17]. Thus, HFIP was used as an additive, and the yield of 2a increased from $45 \%$ to $62 \%$ by addition of HFIP (5 equiv). However, a small amount of the 5-exo product 2a' was obtained (less than $10 \%$, entries $2-4$ ). Furthermore, solvent screening showed that toluene was the best solvent, $\mathrm{CH}_{2} \mathrm{Cl}_{2}$ and $\left(\mathrm{CH}_{2} \mathrm{Cl}\right)_{2}$ also gave the desired product in moderate yields, acetonitrile was less effective, and dimethylformamide (DMF) was ineffective (entries 5-8). Inspired by the results of HFIP, a more acidic additive HOAc was tested. We found that HOAc exhibited similar behavior to HFIP, and the reaction gave product $2 \mathrm{a}$ in $64 \%$ yield. The amount of HOAc affected the yield of product $\mathbf{2 a}$, and 0.6 equiv of HOAc was the best concentration (entries 9-14). Again, the reaction also gave the 5-exo product $\mathbf{2} \mathbf{a}^{\prime}$ in around $5 \%$ yield. It is worth noting that no reaction occurred in the absence of the palladium catalyst (entry 15).

With the optimized reaction conditions, the substrate scope was investigated (Table 2). As with the reaction of 1a (2a 55\% yield, entry 1), the reactions of other substrates with different substituents on the nitrogen also proceeded smoothly. For instance, substrates $\mathbf{1 b}$ and $\mathbf{1 c}$ with $p$-methylbenzyl and $p$-fluorobenzyl substituents gave the corresponding products $\mathbf{2 b}$ and $\mathbf{2 c}$ in moderate yields (55\% and $63 \%$ yield, respectively, entries 2 and 3). Substrate $\mathbf{1 d}$ with the $n$-butyl group gave product 2d in 51\% yield (entry 4).

However, substrate 1e with the phenyl group failed to give the desired product (entry 5). Substrates synthesized from various $\mathrm{N}$-substituted allylamines were also investigated. The reactions also gave the products in moderate yields. For example, substrates $\mathbf{1 f}$ with neopentyl and $\mathbf{1} \mathbf{g}$ with methyl proceed-

\section{Table 1}

Optimization of the reaction conditions.

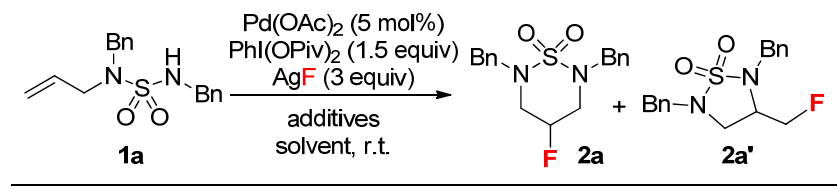

\begin{tabular}{|c|c|c|c|c|}
\hline Entry & Solvent & Additives (equiv) & Yield $(\mathbf{2 a})^{\text {a }}(\%)$ & Yield $\left(\mathbf{2} \mathbf{a}^{\prime}\right)^{\text {a }}(\%)$ \\
\hline 1 & Toluene & - & 45 & 0 \\
\hline 2 & Toluene & $\left(\mathrm{CF}_{3}\right)_{2} \mathrm{CHOH}(1.0)$ & 48 & 6 \\
\hline 3 & Toluene & $\left(\mathrm{CF}_{3}\right)_{2} \mathrm{CHOH}(3.0)$ & 59 & 9 \\
\hline 4 & Toluene & $\left(\mathrm{CF}_{3}\right)_{2} \mathrm{CHOH}(5.0)$ & 62 & 9 \\
\hline 5 & $\mathrm{ClCH}_{2} \mathrm{CH}_{2} \mathrm{Cl}$ & $\left(\mathrm{CF}_{3}\right)_{2} \mathrm{CHOH}(5.0)$ & 42 & 8 \\
\hline 6 & $\mathrm{CH}_{2} \mathrm{Cl}_{2}$ & $\left(\mathrm{CF}_{3}\right)_{2} \mathrm{CHOH}(5.0)$ & 51 & 10 \\
\hline 7 & $\mathrm{CH}_{3} \mathrm{CN}$ & $\left(\mathrm{CF}_{3}\right)_{2} \mathrm{CHOH}(5.0)$ & 22 & 4 \\
\hline 8 & DMF & $\left(\mathrm{CF}_{3}\right)_{2} \mathrm{CHOH}(5.0)$ & - & - \\
\hline 9 & Toluene & HOAc (0.1) & 47 & 5 \\
\hline 10 & Toluene & HOAc (0.5) & 60 & 6 \\
\hline 11 & Toluene & HOAc (0.6) & 64 & 6 \\
\hline 12 & Toluene & HOAc (0.7) & 62 & 6 \\
\hline 13 & Toluene & HOAc (1.0) & 33 & 2 \\
\hline 14 & Toluene & HOAc (2.0) & 19 & 0 \\
\hline $15^{b}$ & Toluene & HOAc (0.6) & 一 & 一 \\
\hline
\end{tabular}

Reaction conditions: 1a (0.1 mmol), [Pd] (5 mol\%), Phl(OPiv) 2 (1.5 equiv), $\mathrm{AgF}$ (3 equiv), additives, solvent ( $0.5 \mathrm{~mL}$ ), at r.t. for $24 \mathrm{~h}$.

a ${ }^{1} \mathrm{H}$ NMR yield with $\mathrm{N}, \mathrm{N}$-dimethyltrifluoroacetamide $\left(\mathrm{CF}_{3}\right.$-DMA) as internal standard. ${ }^{\mathrm{b}}$ Without Pd catalyst. 
Table 2

Substrate scope.

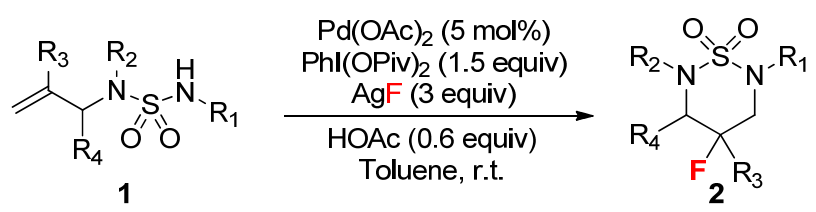

Entry

Reaction conditions: substrate 1 ( $0.2 \mathrm{mmol}$ ), [Pd] (5 mol\%), Phl(OPiv) ${ }_{2}$ (1.5 equiv), AgF (3 equiv), HOAc (0.6 equiv) in toluene (0.5 mL) at r.t. for $24 \mathrm{~h}$ isolated yield.

${ }^{\text {a }}$ Only one isomer was detected.

ed smoothly to give $\mathbf{2 f}$ and $\mathbf{2 g}$ in $76 \%$ and $58 \%$ yields (entries 6 and 7). Substrate $\mathbf{1 h}$ with a phenyl group gave $\mathbf{2 h}$ in $70 \%$ yield (entry 8). Substrate 1i with a tert-butyl acetate group was also compatible with this transformation to give $\mathbf{2 i}$ in $59 \%$ yield (entry 9). For the 1,1-disubstituted substrates, the reaction of $\mathbf{1 j}$ gave product $\mathbf{2 j}$ in $61 \%$ yield (entry 10 ). Furthermore, for substrates with substituents in the allylic position, the reaction exhibited excellent diastereoselectivity to give a single trans-isomer albeit in low yield. The reactions of $\mathbf{1 k}$ and $\mathbf{1 l}$ selectively produced $\mathbf{2 k}$ and $\mathbf{2 l}$ in $37 \%$ and $29 \%$ yields, and the reaction of $\mathbf{1} \mathbf{m}$ generated product $\mathbf{2 m}$ with excellent selectivity and moderate yield (entries 11-13). However, for the trisub- stituted alkene substrate 1n, the reaction did not give the desired product $\mathbf{2 n}$ under the current conditions (entry 14).

Based on our previous results, a plausible mechanism is shown in Scheme 1. The reaction is initiated by nucleopalladation of the alkene to give alkyl-Pd(II) intermediates I and II, which can be oxidized by $\mathrm{PhI}(\mathrm{OPiv})_{2} / \mathrm{AgF}$ to form hypervalent alkyl-Pd-F intermediates [16,17], and the subsequent reductive elimination could address the formation of the C-F bond. Similar to previously reported aminochlorination of alkenes [35], the reaction might involve reversible aminopalladation, and the oxidation of electron-rich intermediate $\mathbf{I}$ is faster than that of intermediate II, resulting in the favorable product being $\mathbf{2 a}$.

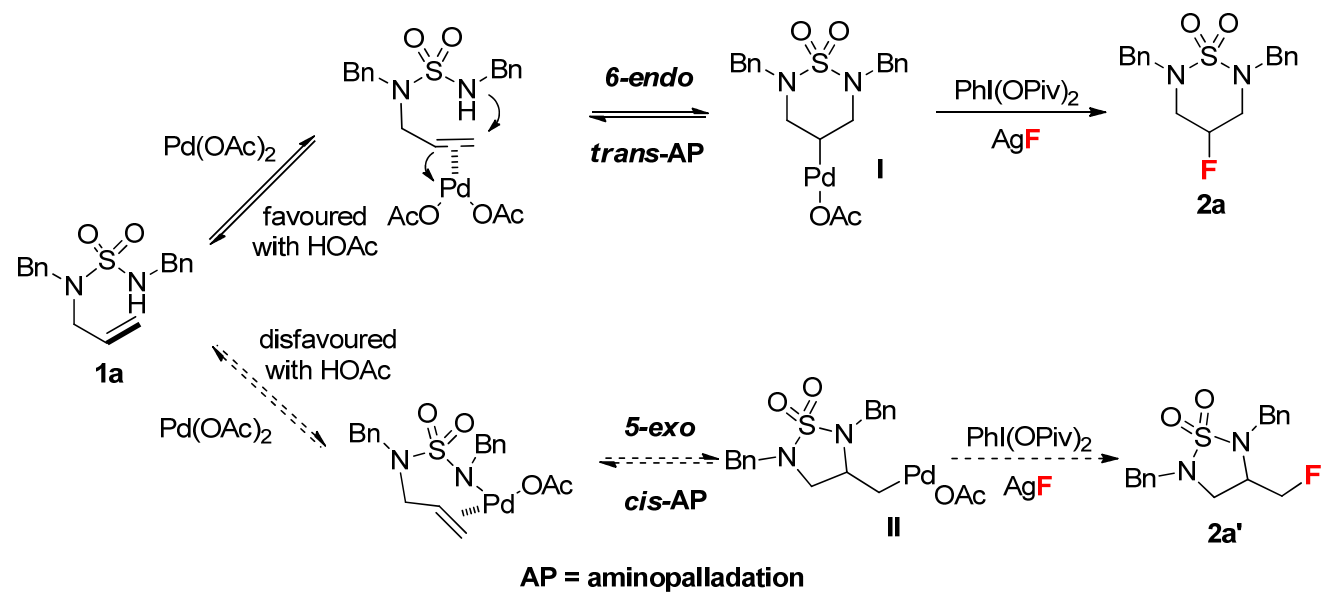

Scheme 1. Proposed mechanism for Pd-catalyzed aminofluorination of unactivated alkenes containing the sulfamide group. 


Graphical Abstract
Chin. J. Catal., 2015, 36: 40-47 doi: 10.1016/S1872-2067(14)60164-9
Pd-catalyzed intramolecular aminofluorination of allylic sulfamides
Jiashun Cheng, Pinhong Chen, Guosheng Liu*
Shanghai Institute of Organic Chemistry, Chinese Academy of Sciences
$\begin{aligned} & \text { A facile Pd-catalyzed intramolecular aminofluorination reaction of unactivated alkenes was developed that can be used to prepare fluori- } \\ & \text { nated 1,3-diamine derivatives. }\end{aligned}$

\section{Conclusions}

In summary, we have developed a novel Pd-catalyzed aminofluorination reaction of unactivated alkenes containing the sulfamide group. The method provides a new approach to synthesize a variety of fluorinated cyclic sulfamide derivatives. HOAc played a crucial role in improving the reactivity to give reasonable yields.

\section{References}

[1] Filler R, Kobayashi Y. Biomedicinal Aspects of Fluorine Chemistry. Amsterdam: Elsevier, 1982.1

[2] Welch J T, Eswarakrishman S. Fluorine in Bioorganic Chemistry. New York: Wiley, 1991. 1

[3] Miyake H. In: Banks R E, Smart B E, Tatlow J C ed. Organofluorine Chemistry: Principles and Commercial Applications. New York: Plenum Press, 1994. 555

[4] Nyffeler P T, Duron S G, Burkart M D, Vincent S P, Wong C H. Angew Chem Int Ed, 2005, 44: 192

[5] Shimizu M, Hiyama T. Angew Chem Int Ed, 2005, 44: 214

[6] Pihko P M. Angew Chem Int Ed, 2006, 45: 544

[7] Petrov V A. Fluorinated Heterocyclic Compounds: Synthesis, Chemistry, and Applications. Hoboken, NJ: John Wiley \& Sons, Inc, 2009

[8] Nosova E V, Lipunova G N, Charushin V N, Chupakhin O N.J Fluorine Chem, 2010, 131: 1267

[9] Zhu S Z, Wang Y L, Peng W M, Song L P, Jin G F. Curr Org Chem, 2002, 6: 1057

[10] Silvester M J. Aldrich Acta, 1991, 24: 31

[11] Kwiatkowski P, Beeson T D, Conrad J C, MacMillan D W C. J Am Chem Soc, 2011, 133: 1738

[12] Kishi Y, Nagura H, Inagi S, Fuchigami T. Chem Commun, 2008: 3876
[13] Fustero S, Catalan S, Sanchez-Rosello M, Simon-Fuentes A, del Pozo C. Org Lett, 2010, 12: 3484

[14] Jensen K H, Sigman M S. Org Biomol Chem, 2008, 6: 4083

[15] Kotov V, Scarborough C C, Stahl S S. Inorg Chem, 2007, 46: 1910

[16] Wu T, Yin G Y, Liu G S. J Am Chem Soc, 2009, 131: 16354

[17] Wu T, Cheng J S, Chen P H, Liu G S. Chem Commun, 2013, 49: 8707

[18] Zhu H T, Liu G S. Acta Chim Sinica (朱海涛, 刘国生. 化学学报), 2012, 70: 2404

[19] Qiu S F, Xu T, Zhou J, Guo Y L, Liu G S. J Am Chem Soc, 2010, 132: 2856

[20] Xu T, Qiu S F, Liu G S. Chin J Chem (徐涛, 邱水发, 刘国生. 中国化 学), 2011, 29: 2785

[21] Xu T, Mu X, Peng H H, Liu G S. Angew Chem Int Ed, 2011, 50: 8176

[22] Xu T, Liu G S. Org Lett, 2012, 14: 5416;

[23] Liu Q L, Wu Y C, Chen P H, Liu G S. Org Lett, 2013, 15: 6210

[24] Qian J Q, Liu Y K, Zhu J, Jiang B, Xu Z Y. Org Lett, 2011, 13: 4220

[25] Li Z D, Song L Y, Li C Z. J Am Chem Soc, 2013, 135: 4640

[26] Wang Q Zhong W H, Wei X, Ning M H, Meng X B, Li Z J. Org Biomol Chem, 2012, 10: 8566

[27] Huang H T, Lacy T C, Blachut B, Ortiz Jr G X, Wang Q. Org Lett, 2013, 15: 1818

[28] Cui J, Jia Q, Feng R Z, Liu S S, He T, Zhang C. Org Lett, 2014, 16: 1442

[29] Kong W Q Feige P, de Haro T, Nevado C. Angew Chem Int Ed, 2013, 52: 2469

[30] Shuntona H P, Früeh N, Wang Y M, Rauniyar V, Toste F D. Angew Chem Int Ed, 2013, 52: 7724

[31] Eickmeier C, Peters S, Fuchs K, Heine N, Handschuh S, DornerCiossek C, Klinder K, Kostka, M. US Patent 5006 939. 2006

[32] Martinez A, Castro A, Gil C, Miralpeix M, Segarra V, Domenech T, Beleta J, Palacios J M, Ryder H, Miro X, Bonet C, Casacuberta J M, Azorin F, Pina B, Puigdomenech P. J Med Chem, 2000, 43: 683

[33] Armarego W L F. Purification of Laboratory Chemicals. Amsterdam: Elsevier, 1997

[34] McDonald R I, Stahl S S. Angew Chem Int Ed, 2010, 49: 5529

[35] Yin G Y, Wu T, Liu G S. Chem Eur J, 2012, 18: 451

\title{
钯催化烯丙基磺酰胺的分子内胺氟化反应
}

\author{
程家顺, 陈品红, 刘国生* \\ 中国科学院上海有机化学研究所金属有机国家重点实验室, 上海200032
}


环合产物, 实现了氟代的环状磺胺类产物的有效合成.

关键词：钯催化; 胺氟化; 烯烃; 氟代1,3-二胺; 6-endo环合

收稿日期: 2014-05-19. 接受日期: 2014-06-09. 出版日期: 2015-01-20.

*通讯联系人.电话: (021)54945346; 传真: (021)64166128; 电子信箱: gliu@mail.sioc.ac.cn

基金来源：国家重点基础研究发展计划(973计划, 2011CB808700); 国家自然科学基金(21225210, 20923005和21121062); 上海市 科学技术委员会(11JC1415000); 中国科学院、国家外国专家局创新团队国际合作伙伴计划.

\section{1. 前言}

在有机分子中引入氟原子能够极大地改变它们的 性能, 如溶解性、代谢稳定性以及生物药效率 ${ }^{[1,2]}$. 在这 些有机氟化物中, 氟代杂环作为重要的合成片段已被用 于合成抗胆碱能药、止吐剂、镇痉剂以及一些酶抑制剂 等 ${ }^{[3]}$. 因此, 如何有效地合成这些氟代杂环引起了广泛 地关注 ${ }^{[4-13]}$.

钯催化烯烃的双官能团化反应是直接将简单的烯 烃原料转化为邻位双取代分子的一种快速有效的途径. 这些反应中, 烷基钯中间体的氧化对双官能团化反应极 为重要, 反应通过烯烃的亲核钯化启动产生的烷基钯中 间体, 最终碳-杂键的形成推测是经过高价钯络合物的 还原消除 ${ }^{[14,15]}$. 在2009年, 我们小组报道了首例钯催化 非活性烯烃的分子内胺氟化反应, 推测反应在氟化银/硾 苯季戊酸 $\left(\mathrm{AgF} / \mathrm{PhI}(\mathrm{OPiv})_{2}\right)$ 条件下, 通过烷基碳-钯键的 氧化断裂来形成碳-氟键; 该反应可以用于高区域选择 性地合成一系列氟代哌啶类化合物 ${ }^{[15]}$. 近期, 我们发现 通过改变氮原子上的保护基可以完全改变反应的区域 选择性, 有效地合成一系列含一氟亚甲基的四氢吡咯类 化合物 ${ }^{[17]}$. 利用该催化体系, 我们还实现了苯乙烯的分 子间胺氟化反应 ${ }^{[18]}$. 同时, 我们组在钯催化下, 利用 $\mathrm{N}-$ 氟代双苯环酰亚胺(NFSI)作为氮源和氟源, 也实现了苯 乙烯的分子间胺氟化反应 ${ }^{[19,20]}$. 自此, 烯烃的胺氟化反 应得到了广泛的研究. Li等 ${ }^{[25]}$ 报道了利用Selectfluor作 为氟化试剂, 银催化的非活性烯烃的胺氟化反应. $\mathrm{Li}$ 等 ${ }^{[26] 、 W a n g}$ 等 ${ }^{[27]}$ 和Zhang等 ${ }^{[28]}$ 分别报道了非金属参与的 分子内烯烃的胺氟化反应; 相应的不对称反应则由 Nevado等 ${ }^{[29]}$ 和Toste等 ${ }^{[30]}$ 进行了报道. 鉴于环状磺酰胺 在药物化学中的重要价值 ${ }^{[31,32]}$, 我们希望发展合成这一 类氟代杂环的有效方法, 其中通过钯催化烯丙基磺酰胺 的氟胺化反应可能是合成这一类化合物的最有效的方 法之一. 在此, 本文针对该工作进行报道.

\section{2. 实验部分}

\section{1. 实验通则}

所有商品化的化合物都是购买后直接使用; ${ }^{1} \mathrm{H}$ 和 ${ }^{13} \mathrm{C} N M R$ 谱通过安捷伦- $400 \mathrm{MHz}$ 测试, 以気代氯仿 $\left(\mathrm{CDCl}_{3}\right)$ 作为溶剂, 分别以 TMS $(0 \mathrm{ppm})$ 作为 ${ }^{1} \mathrm{H}$ 内标, $\mathrm{CDCl}_{3}(77.0 \mathrm{ppm})$ 作为 ${ }^{13} \mathrm{C}$ 内标. 快速柱层析通过硅胶柱 (200-400目, 中国烟台), 以石油醚/乙酸乙酯作为洗脱剂. 所有溶剂根据文献[33]进行干燥和纯化. $\mathrm{CDCl}_{3}$ 购买于 $\mathrm{J} \& \mathrm{~K}$ (99.8 atom\% D, 上海, 中国), $\mathrm{AgF}$ 购买于Aldrich (99.9\%, 上海, 中国), 六氟异丙醇 (HFIP) 购买于 $\mathrm{TCI}$ (99.0\%, 上海, 中国), 底物1a-1n根据文献[34]步骤合成.

\section{2. 氟胺化反应}

将 $\mathrm{Pd}(\mathrm{OAc})_{2}$ (2.2 mg, $\left.0.01 \mathrm{mmol}\right), \mathrm{AgF}$ (76.2 mg, 0.6 $\mathrm{mmol}), \mathrm{PhI}(\mathrm{OPiv})_{2}(121.8 \mathrm{mg}, 0.3 \mathrm{mmol})$ 和底物烯烃 $(0.2$ $\mathrm{mmol})$ 加入干燥的玻璃管中, 加入醋酸 $(120 \mu \mathrm{L}, 1 \mathrm{~mol} / \mathrm{L}$, $0.12 \mathrm{mmol})$ 的甲苯溶液 $(1 \mathrm{~mL})$. 在室温下搅拌 $24 \mathrm{~h}$ 后过 滤, 固体用乙酸乙酯洗涤, 滤液真空浓缩后通过快速柱 层析, 以石油醚/乙酸乙酯作为洗脱剂, 纯化得到产物.

\section{3. 结果与讨论}

我们首先以烯丙基磺酰胺 $1 \mathrm{a}$ 为底物, 采用之前的胺 氟化反应条件 ${ }^{[17]}$ 进行探索反应, 以 $45 \%$ 的收率高区域选 择性地得到胺氟化产物 $2 \mathbf{a}$ (表 1 , 实验 1 ). 在我们之前的 研究中, 加入酸性添加剂, 如六氟异丙醇(HFIP)有利于 提高胺氟化反应的物料平衡 ${ }^{[17]}$. 因此, 我们在体系中加 入 HFIP 作为添加剂, 当HFIP的添加量为 5 当量时, 产物 $2 \mathrm{a}$ 的收率由 $45 \%$ 提高至 $62 \%$, 但是反应伴随着少量 5-exo 产物 $2 \mathbf{a}^{\prime}$ 生成, 收率小于 $10 \%$ (实验2-4). 通过溶剂篎选表 明甲苯是最佳溶剂, 二氯甲烷和二氯乙烷中也能以中等 收率得到目标产物, 但是乙腈效果很差, 在 $N, N$-二甲基 甲酰胺(DMF)中, 反应没有产物(实验5-8). 根据HFIP的 结果, 我们尝试了酸性更强的醋酸作为添加剂. 我们发 现与六氟异丙醇类似, 醋酸也能促进该反应, 产物 $\mathbf{2 a}$ 收 率达 $64 \%$. 其中醋酸的添加量也对产物收率有明显影 响, 以 0.6 当量为最佳, 但反应仍然有 $5 \%$ 的 5-exo产物 $2 \mathbf{a}^{\prime}$ (实验9-14). 值得注意的是, 在没有钯催化剂的条件 下, 反应是不能发生的(实验 15 ).

在上述最优条件下, 我们考察了该反应的底物普适 
性(表2), 与底物 $\mathbf{1 a}$ 相比(生成 $\mathbf{2 a}$, 收率 $55 \%$, 实验 1 ), 其它 氮原子上含有不同取代基的底物也能很好地进行该反 应. 例如, 含对甲基苠基底物 $1 \mathrm{~b}$ 和对氟苠基底物 $1 \mathrm{c}$ 分别 以中等的收率得到相应的 $\mathbf{2 b}$ 和 $\mathbf{2 c}$ (收率分别为 $55 \%$ 和 $63 \%$, 实验 2 和 3 ). 正丁基取代的底物 $1 \mathrm{~d}$ 以 $51 \%$ 的收率得 到产物 2d (实验4), 但是苯基取代的底物 1e在标准条件 下无法得到目标产物(实验5)。随后考察了氮上不同取 代的烯丙基胺底物, 例如氮上新戊基取代的底物 $\mathbf{1 f}$ 和甲 基取代的 $1 \mathrm{~g}$ 都能顺利进行，分别以 76\%和 58\%的收率得 到产物 $2 \mathrm{f}$ 和 $2 \mathrm{~g}$ (实验 6 和 7 ), 氮上苯基取代的底物 $1 \mathrm{~h}$ 能以 $70 \%$ 的收率给出目标产物 $\mathbf{2 h}$ (实验 8 ). 含叔丁酯的底物 $1 \mathbf{i}$ 也能在该催化体系中兼容, 目标产物 $\mathbf{2 i}$ 的收率为 $59 \%$ (实验9). 我们也考察了 $1,1-$ 双取代的烯烃 $\mathbf{j} \mathbf{j}$, 相应产物 $\mathbf{2} \mathbf{j}$ 的收率为 $61 \%$ (实验 10 ). 此外, 对于烯丙基链上有取代 基的底物, 反应能以优秀的非对映选择性得到单一的反 式产物, 但是产率稍低; 比如底物 $1 \mathrm{k}$ 和 11 生成目标产物 $\mathbf{2 k}$ 和 21 的收率分别为 $37 \%$ 和 $29 \%$, 但是产物 $\mathbf{2 m}$ 的收率可 达中等(实验11-13). 然而, 对于三取代的烯烃 1n, 在标 准条件下未能生成产物(实验14).

基于以上结果, 我们推测反应可能机理如下(图式 1 ): 反应首先通过烯烃的亲核钯化启动得到烷基钯中间 体I和II, 接下来被 $\mathrm{AgF} / \mathrm{PhI}(\mathrm{OPiv})_{2}$ 氧化到高价烷基钯氟 中间体 ${ }^{[16,17]}$, 紧接着还原消除形成 $\mathrm{C}-\mathrm{F}$ 键. 与我们之前 报道的烯烃的胺氯化反应结果类似 ${ }^{[35]}$, 该反应也可能经 历一个可逆的胺钯化, 其中相对富电子的中间体 I的氧 化远远快于中间体II, 因此, 反应有利于生成产物 $2 \mathbf{a}$.

\section{4. 结论}

我们发展了一种新颖的钯催化非活性烯烃的胺氟 化反应, 所采用的烯烃含有磺酰胺官能团, 该方法为合 成一系列氟代的环状磺酰胺衍生物提供了新的途径. 醋 酸的添加有利于提高反应活性, 生成产物的收率较高. 